\title{
Improving Detectors for Cryo-Electron Microscopy
}

\author{
Paul Mooney ${ }^{1}$ \\ 1. Gatan, Inc. Pleasanton, CA 94588.
}

Cryo-electron microscopy places a number of demands on TEM image recording performance. The need to capture specimen information before it is destroyed by beam damage requires the highest possible detective quantum efficiency (DQE) ${ }^{1}$. Further, the newly understood issue of beam-induced motion ${ }^{2}$ and its mitigation by dose-fractionated motion correction ${ }^{3}$ mean DQE must remain high at dose levels $<1 \mathrm{e}^{-/}$pixel and that the camera should have a high frame-rate to track the motion. Pixel count is also important in determining the accuracy of frame alignment and for providing throughput. But DQE is still the core issue, so physical processes which reduce DQE and the measures which have been taken to mitigate them will be the focus of what follows.

The biggest issue affecting DQE is elastic electron scattering: 1) in the detection medium itself, 2 ) in any associated supporting layer and 3) in the surroundings. Even after the advent of digital cameras which could provide immediate output, digitized film remained the recording medium of choice. This may have been in part due to the transmission geometry of film which reduced the number of scattered electrons. However, the achievement of high resolution was limited primarily to the domain of high-symmetry virus capsid structures which, due to their large size, could be found and aligned more easily. All agreed there was a need for a better detector that could also show the results immediately.

The first fiber optically coupled scintillator/CCD cameras were greeted with great interest by the cryo-EM community but electron scatter from the fiber optic substrate prevented their immediate adoption. The issue was addressed partially though an enlargement of the camera from $19 \mu \mathrm{m} 1 \mathrm{k}$ x $1 \mathrm{k}$ (the Gatan SSC $^{\mathrm{TM}}$ and MSC ${ }^{\mathrm{TM}}$ ) to $24 \mu \mathrm{m} 2 \mathrm{k} \mathrm{x} 2 \mathrm{k}$ (the Gatan MegaScan ${ }^{\mathrm{TM}}$ ). The latter was successful at generating the high DQE both at low spatial frequency and at the very low doses which were needed for electron diffraction. The MegaScan's larger detector enabled a hybrid data-taking approach in electron crystallography with 2D crystal images still acquired by film ${ }^{4}$. In the $1 \mathrm{k} \mathrm{x} 1 \mathrm{k}$ format, the same DQE at low dose with the camera's immediate readout capability provided the platform for the development of tomography automation ${ }^{5}$. The Gatan UltraScan ${ }^{\mathrm{TM}}$, with a further increase in size and finer sampling $(15 \mu \mathrm{m}$ pixel $4 \mathrm{k} \mathrm{x} 4 \mathrm{k})$ and faster readout, made possible the integrated automation of single-particle acquisition for intermediate resolution projects 6 .

In the late 1990 s and early 2000 s, various potential solutions to the scattering problem were explored: use of a fiber-optic taper to enlarge the effective pixel size ${ }^{7}$; use of a directlyilluminated hybrid pixel detector to increase pixel size and sensitivity ${ }^{8}$; reducing the size of the electron interaction volume through deceleration ${ }^{9}$ and recreating a transmission medium with a low-backscatter mirror, large lens and back-illuminated CCD. The last instance, labelled the UltraCam $^{\mathrm{TM}}$, nearly eliminated elastic scattering from the electron interaction, thereby greatly improving intermediate frequency DQE. But the low spatial frequency DQE didn't go up from the level of a fiber optically coupled CCD camera as expected ${ }^{10}$, exposing the second key noise source: the fluctuations in the energy deposited by inelastic scattering in a thin detector. It may have been that a second advantage of film was that its quasi-digital grain exposure mechanism 
acted as a discriminator, reducing the effects of thin-detector energy deposition fluctuations. The lens-coupled UltraCam ${ }^{\mathrm{TM}}$ unfortunately did not have a strong enough signal to allow a similar kind of discrimination.

Fortunately, at this time, investigations were also underway using CMOS active pixel sensors (APS) for TEM electron detection ${ }^{11}$. These sensors provided the means to address both elastic and inelastic modes of noise formation. An APS can be thinned to operate in a transmission mode, virtually eliminating the effects of elastic scattering. It also has sufficient secondary electron yield to allow event discrimination for the mitigation of inelastic fluctuations.

Discrimination requires that events created by incoming electrons be spaced far enough apart. Since beam sparsification requires very high speed readout, a compromise approach has been implemented in one type of camera which optimizes the thickness of the electron-sensitive layer such that elastic scattering and inelastic fluctuations are balanced and the resulting noise is minimized $^{12}$. Another implementation of CMOS APS, the Gatan K2 camera, provides a high frame-rate (400fps) to sparsify the beam at detector dose rates of 1-10 e-/pixel/s and includes hardware for discrimination, centroiding and counting of the events at that frame-rate. Both approaches have now been shown better than film both in DQE and in the ability to manage beam-induced motion. The discrimination (or counting) approach has been shown to open up a new range of resolution ${ }^{13}$, has enabled study of smaller molecules ${ }^{13}$ and has improved handling of conformational heterogeneity ${ }^{14}$. Given the results achieved so far with counting, it is anticipated that continued improvements to detector hardware will contribute to further significant advances in cryo-EM.

\section{References:}

[1] de Ruijter, W.J., Micron 26 (1995), pp. 247-275.

[2] Brilot, A.F. et al, Journal of Structural Biology 177 (2012), pp. 630-667.

[3] Li, X. et al, Nature Methods 10 (2013), pp. 584-590.

[4] Downing, K. and Hendrickson, F., Ultramicroscopy 75 (1999), p. 215-233.

[5] Koster, A.J. et al, Ultramicroscopy 46 (1992), pp. 207-227.

[6] Zhang, P. et al, Journal of Structural Biology 143 (2003), pp. 135-144.

[7] Faruqi, A.R. et al, Ultramicroscopy 75 (1999), pp. 235-250.

[8] Faruqi, A.R. and Cattermole, D.M., Nuclear Instr. And Meth. A 549 (2005), pp. 192-198.

[9] Downing, K.H. and Mooney, P.E., Review of Scientific Instruments 79 (2008).

[10] Bailey, B. et al, Microscopy and Microanalysis 10 (2004), pp. 1204-1205.

[11] Battaglia, M. et al, Nuclear Instr. And Meth. A 598 (2009), pp. 642-649.

[12] Clough, R. and Kirkland, A.J., Advances in Imaging and Electron Physics 198 (2016), ed. Hawkes, P.W., p. 32.

[13] Merk, A. et al, Cell 165 (2016), pp. 1-10.

[14] Yan, C. et al, Science (2015), pp. 1182-1191. 